\title{
Ultrasonography Misdiagnosis of Postmenopausal Endometrial Tuberculosis (PET) Caseous Necrosis: A Case report
}

\section{Arzigul Umarjan, Xiang Hong* and Hu Rong}

Department of Ultrasound Diagnosis, Obstetrics and Gynecology of First Affiliated Hospital of Xinjiang Medical University, Xinjiang, PR china

*Corresponding author: Xiang Hong, Department of Ultrasound Diagnosis, Obstetrics and Gynecology of First Affiliated Hospital of Xinjiang Medical University, Xinjiang, PR china, Tel: +86 13579970943, E-mail: xianghong65@126.com

Received date: February 10, 2017; Accepted date: March 31, 2017; Published date: April 05, 2017

Citation: Umarjan A, Hong X, Rong H (2017) Ultrasonography Misdiagnosis of Postmenopausal Endometrial Tuberculosis (PET) Caseous Necrosis: A Case report. Reproductive Immunol Open Acc 2: 29. doi: 10.21767/2476-1974.100029

Copyright: (c) 2017 Umarjan A, et al. This is an open-access article distributed under the terms of the Creative Commons Attribution License, which permits unrestricted use, distribution, and reproduction in any medium, provided the original author and source are credited.

\section{Abstract}

Objective: To reduce misdiagnosis and not misdiagnose of postmenopausal endometrial tuberculosis.

Method: Analyzing a case of postmenopausal endometrial tuberculosis caseous necrosis misdiagnosed as endometrial cancer by ultrasonography.

Result: This case was clearly diagnosed as postmenopausal endometrial tuberculosis by means of hysteroscopy curettage and pathological examination, thereby the misdiagnosis was rectified.

Conclusion: Ultrasonography has significant implications for clinical diagnosis of postmenopausal caseous endometrial tuberculosis and identification of endometrial cancer. It is suggested to carry out clinical curettage and pathological examination in time so as to reduce misdiagnosis of postmenopausal endometrial tuberculosis.

\section{Clinical Data}

Patients, female, 65 years old, menopause for 8 years, lower abdominal pain for more than 6 months to add up to 20 days, to our hospital for treatment. Patients complains that no history of tuberculosis, and nearly half a year to lose weight $5 \mathrm{~kg}$ By transvaginal ultrasound examination, a heterogeneity echo area of about $5.1 \times 4.8 \times 7.3 \mathrm{~cm}$ (normal mesometrium peripheral is about $0.44 \mathrm{~cm}$ thick) could be seen in the middle part of the uterus, and an irregular echo-free area of about $2.5 \times 1.3 \times 5.6$ could be seen in the middle part with poor sound transmission and floating flocculent middle-low echo visible, enveloped by hypoecho. The lining is obscure. CDFI: obvious bleeding signal is unseen (Figures $A, B, C$ and $D$ ) and nothing was injected to outline. Bilateral ovaries are obscure; bilateral adnexa areas have no obvious abnormal sign. Heterogeneity echo is hinted in the uterine cavity. Given considerations to the possibility of endometrial cancer combined with endometrial cavity fluid; other possibility could not be excluded, and further examination is suggested. Then, hysteroscopy curettage was conducted, and the pathological examination result indicated that the substance in the uterus was plenty of caseous necrosis and granuloma lesion, identified as tuberculosis in histomorphology. The patient was transferred to the specialized department and received antituberculosis therapy; two months later, her symptoms relieved.

\section{Discussion}

Among tuberculosis in all parts of the body, genital tuberculosis ranks only second to phthisis; and the most frequent part of genital tuberculosis is the fallopian tube (95\%-100\%), followed by uterus (50\%-60\%), ovary (20\%-30\%), cervix (5\%-15\%) [1]. Endometrial tuberculosis, mostly menstruation infection, reaches the basal layer of the lining via the fallopian tube in non-menstruation, and may cause repeated endometrial infection when flowing with menstrual blood in menstruation [2]. Female genital tuberculosis mostly strikes women aged $20 \sim 40$ in child-bearing period, and postmenopausal endometrial tuberculosis is rarely seen, therefore, it is easy to be misdiagnosed as endometrial cancer frequently [3]. Barbour and Towers believe that after menopause the uterine lining stops shedding periodically, therefore, curettage of postmenopausal endometrial tuberculosis is applied more widely than child-bearing age. They are mostly caseous lesions, about $60 \%-70 \%$ of the endometrium is invaded [4,5]. Postmenopausal women have low immunity while the dormant bacteria have powerful virulence. The lesion behaves as vaginal fluid mainly by exudation [6]. The abdominal pain is concerned with the mechanical stimulation caused by the plenty of endometrial cavity fluid and cervical adhesion generated by inflammatory stimulation and lesion [7]. The clinical symptoms of endometrial tuberculosis have no specificity, and the ultrasonography manifestation of postmenopausal endometrial tuberculosis is also short of specificity, and its ultrasonoscopy is similar to endometrial cancer, so it is easy to be misdiagnosed as endometrial cancer. The special pathological changes of tuberculosis result in large variation of the ultrasonic image: at early phase, it manifests irregular thickness of endometrium and strong echo, and the lining is serrated against the endometrial cavity fluid. Hereafter, 
the endometrium necroses, sticks together and becomes calcified; and on the ultrasonoscopy, the uterine cavity has strong limited massive echo or strong echo spots accompanied by acoustic shadow in the rear. On the ultrasonoscopy, irregular thickness of endometrium is visible, no CDFI or a little bleeding signal and endometrial cavity fluid triad [8]. The possibility of endometrial tuberculosis should be given paramount consideration. Ultrasonography is noninvasive and applied widely with high accuracy rate, so it can be used as the first choice for examining endometrial tuberculosis. In this case, the patient's ultrasonoscopy manifests abnormal endometrial echo. Although the patient is menopausal, tuberculosis has a high incidence in developing countries [9]. For fear of misdiagnosis or missed diagnosis, extra vigilance should be attached to tuberculosis. It is important to come up with the possibility of tuberculosis when diagnosing. And checked the patient's history of pulmonary TB as well as her sex partner to investigate the source of infection.

Ultrasonography has significant implications for clinical diagnosis of postmenopausal caseous endometrial tuberculosis and identification of endometrial cancer. It is suggested that clinical curettage is a fatal mistake; because it may spread the infection in the uterus and hematogenus spread of infection may occur.

Maybe fine needle aspiration could be a better way to collect samples for lab. Diagnosis, the anti-tuberculosis therapy should be started before clinical curettage to avoid spreading of infection. Moreover in clinical diagnosis, the doctor shall inquiry the patient about medical history in detail, make comprehensive analysis on medical history, symptoms, signs and each auxiliary examination, fully take the possibility of tuberculosis into consideration and carry out each examination on tuberculosis so as to reduce misdiagnosis rate.
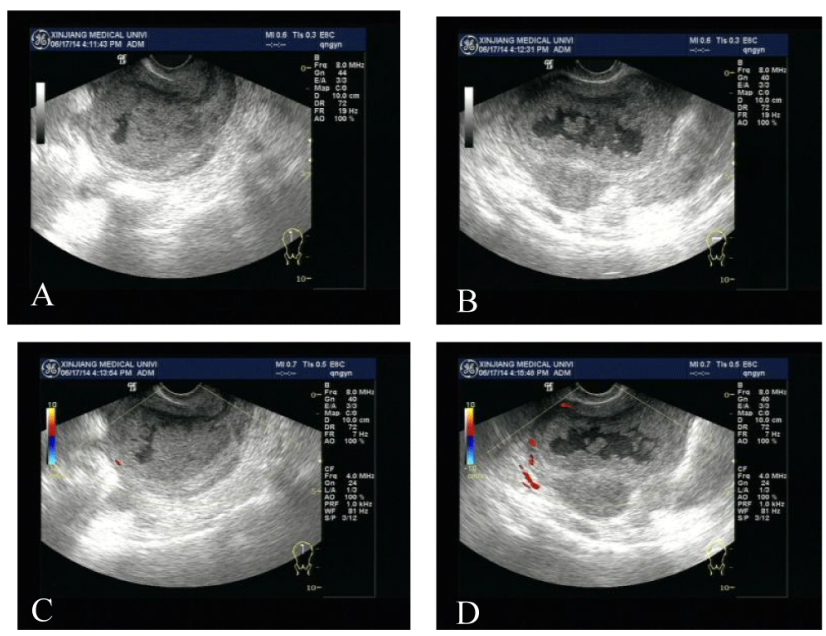

Figures A-D: Transvaginal ultrasound examination

\section{References}

1. Zhangxiaodan (2008) Discussion on Female Genital Tuberculosis Control. World Health Digest 3.

2. Deng qiao zi, Liu xiao hong, Wang yan (2005) Analysis of 37 cases of female genital tuberculosis misdiagnosed. Chinese J Misdiag 15: 8.

3. Lu xue-mei, Peng yan-li, Gao jing-ru (2015) Preliminary Study of the Clinical Diagnosis on Postmenopausal Endometrial Tuberculosis. Chinese Journal of Medicinal Guide 17: 662

4. Towers RP (1954) Post-menopausal endometrial tuberculosis; an unusual case with a review of previous reports. J Obstet Gynaecol Br Emp 61: 657-660.

5. Brien FS (1962) Tuberculosis: a challenge to the general practitioner. Can Med Assoc J 87: 1326-1331.

6. Maestre MA, anzano CDLopez RM (2004)Postmenopausal endometrial tuberculosisInt J Gynaecol Obstet 86: 405-406.

7. Lamb J, Eckert L, Deubner H (2004) Postmenopausal endometrial tuberculosis: a case report. Infect Dis Obstet Gynecol 1: 2.

8. Wen-juan MAO, Li-qun CHEN, Hong SUN (2012) CDFI Blood Flow Analysis in Diagnosis of Postmenopausal Endometrial Thickening. Chinese Computed Medical Imaging 18: 3.

9. Cohn DL, Bustreo F, Raviglione MC (1997) Drug-Resistant Tuberculosis: Review of the Worldwide Situation and the WHO/ IUATLD Global Surveillance Project. Clin Infect Dis 24: 121-130. 